\title{
Facial parity edge colouring
}

\author{
Július Czap
}

Department of Applied Mathematics and Business Informatics, Faculty of Economics, Technical University of Košice, B. Němcovej 32, 04001 Košice, Slovakia and Institute of Mathematics, Faculty of Science, Pavol Jozef Šafárik University Jesenná 5, 04001 Košice, Slovakia

Stanislav Jendrol'

Institute of Mathematics, Faculty of Science, Pavol Jozef Šafárik University Jesenná 5, 04001 Košice, Slovakia

\section{František Kardoš}

Institute of Mathematics, Faculty of Science, Pavol Jozef Šafárik University Jesenná 5, 04001 Košice, Slovakia

and

Institute for Theoretical Computer Science (ITI), Faculty of Mathematics and Physics, Charles University, Malostranské Náměstí 25, 11800 Prague, Czech Republic

Received 10 December 2009, accepted 23 December 2010, published online 26 May 2011

\begin{abstract}
A facial parity edge colouring of a connected bridgeless plane graph is an edge colouring in which no two face-adjacent edges (consecutive edges of a facial walk of some face) receive the same colour, in addition, for each face $\alpha$ and each colour $c$, either no edge or an odd number of edges incident with $\alpha$ is coloured with $c$. From Vizing's theorem it follows that every 3 -connected plane graph has a such colouring with at most $\Delta^{*}+1$ colours, where $\Delta^{*}$ is the size of the largest face. In this paper we prove that any connected bridgeless plane graph has a facial parity edge colouring with at most 92 colours.
\end{abstract}

Keywords: plane graph, facial walk, edge colouring

Math. Subj. Class.: 05C10, 05C15

E-mail addresses: julius.czap@tuke.sk, julius.czap@upjs.sk (Július Czap), stanislav.jendrol@upjs.sk (Stanislav Jendrol'), frantisek.kardos@upjs.sk (František Kardoš) 


\section{Introduction}

One of the motivations for this paper has come from recent papers of Bunde et al. [1, 2] who introduced parity edge colourings of simple graphs. Studying the parity of the usage of colours along walks suggested two edge-colouring parameters that have interesting properties and applications. A parity walk in an edge colouring of a simple graph is a walk along which each colour is used an even number of times. Bunde et al. [2] introduced two parameters. Let $p(G)$ be the minimum number of colours in an edge colouring of $G$ having no parity path (a parity edge colouring). Let $\widehat{p}(G)$ be the minimum number of colours in an edge colouring of $G$ in which every parity walk is closed (a strong parity edge colouring). Since incident edges of the same colour would form a parity path of length 2 , every parity edge colouring is a proper edge colouring, and hence $p(G) \geq \chi^{\prime}(G)$, where $\chi^{\prime}(G)$ is the chromatic index of $G$. Since a path is an open walk, no strong parity edge colouring has a parity path. Hence, every strong parity edge colouring is a parity edge colouring and $\widehat{p}(G) \geq p(G)$ for every graph $G$. Although there are graphs $G$ with $\widehat{p}(G)>p(G)$ [2], it remains unknown how large $\widehat{p}(G)$ can be when $p(G)=k$. Elementary results on these parameters appear in [2]. In [1] it is proved that $\widehat{p}\left(K_{n}\right)=2^{\lceil\log (n)\rceil}-1$ for all $n$. Moreover, the optimal strong parity edge colouring of the complete $n$-vertex graph $K_{n}$ is unique when $n$ is a power of 2. The authors of [2] mentioned that computing $p(G)$ or $\widehat{p}(G)$ is NP-hard even when $G$ is a tree. Clearly, the parity edge colouring is such a colouring that each path uses at least one colour an odd number of times.

The vertex version of this problem (strong parity vertex colouring) with some restrictions was introduced in [3]. The authors of [3] conjectured that there is a constant $K$ such that the vertices of any 2-connected plane graph can be coloured with at most $K$ colours in such a way that for each face $\alpha$ and each colour $c$, either no vertex or an odd number of vertices incident with $\alpha$ is coloured with $c$.

Another motivation for this work has come from the papers of Pyber [5] and Mátrai [4]. A graph is called odd if the degree of its vertices is odd or zero. Pyber raises the problem of edge covering with odd subgraphs in [5] as the counterpart of even subgraph covering problems. He proved that the edges of every finite simple graph can be covered by at most 4 edge-disjoint odd subgraphs; moreover, if the number of vertices is even then 3 odd subgraphs are sufficient. For not necessarily edge-disjoint coverings we have the following question: Is it true that every graph can be covered by at most 3 odd subgraphs? Mátrai in [4] showed that every finite simple graph can be covered by 3 odd subgraphs and he found an infinite sequence of finite simple connected graphs not coverable by 3 edge-disjoint odd subgraphs.

Pyber's result implies the following: The edges of any 3-connected plane graph $G$ can be coloured by at most 4 colours in such a way that for each face $\alpha$ and each colour $c$, either no edge or an odd number of edges incident with $\alpha$ is coloured with $c$. It is sufficient to consider the dual $G^{*}$ of $G$ and its edge cover with at most 4 edge-disjoint odd subgraphs. This cover induces the required colouring of $G$.

If we add a requirement that such a colouring must be proper, then it is not clear whether there exists a colouring with $K$ colours, where $K$ is an absolute constant. From Vizing's theorem [6] it follows that every 3 -connected plane graph $G$ has such a colouring which uses at most $\Delta^{*}+1$ colours, where $\Delta^{*}$ is the size of the largest face. Consider a proper edge colouring of the dual graph $G^{*}$. This colouring induces a colouring of $G$ in a natural way. It is such a colouring in which, for each face $\alpha$ of $G$, all the edges in the boundary of $\alpha$ have distinct colours. 
In this paper we show that each connected bridgeless plane graph has a facial parity edge colouring using at most 92 colours.

\section{Notation}

Let us introduce the notation used in this paper. A graph which can be embedded in the plane is called planar graph; a fixed embedding of a planar graph is called plane graph.

A bridge is an edge whose removal increases the number of components. A graph which contains no bridge is said to be bridgeless. In this paper we consider connected bridgeless plane graphs, multiple edges and loops are allowed.

Let $G=(V, E, F)$ be a connected plane graph with the vertex set $V$, the edge set $E$, and the face set $F$. The degree of a vertex $v$, denoted by $\operatorname{deg}(v)$, is the number of edges incident with $v$, each loop counting as two edges. A $k$-vertex is a vertex of degree $k$. For a face $\alpha$, the size of $\alpha, \operatorname{deg}(\alpha)$, is defined to be the length of its facial walk, i.e. the shortest closed walk containing all edges from the boundary of $\alpha$. We often say $k$-face for a face of size $k$.

Given a graph $G$ and one of its edges $e=u v$ (the vertices $u$ and $v$ do not have to be different), the contraction of $e$ denoted by $G / e$ consists of replacing $u$ and $v$ by a new vertex adjacent to all the former neighbours of $u$ and $v$, and removing the loop corresponding to the edge $e$. (We keep multiple edges if they arise.) Analogously we define the contraction of the set of edges $H=\left\{e_{1}, \ldots, e_{k}\right\}$ and we denote it by $G /\left\{e_{1}, \ldots, e_{k}\right\}$ or $G / H$.

Two faces are adjacent if they share an edge. Two (distinct) edges are face-adjacent if they are consecutive edges of a facial walk of some face $\alpha$.

A $k$-edge colouring of a graph $G$ is a mapping $\varphi: E(G) \rightarrow\{1, \ldots, k\}$. The facial parity edge (FPE) colouring of a connected bridgeless plane graph is such an edge colouring that no two face-adjacent edges receive the same colour, in addition, for each face $\alpha$ and each colour $c$, either no edge or an odd number of edges incident with $\alpha$ is coloured with $c$.

Question 2.1. What is the minimum number of colours $\chi_{f p}^{\prime}(G)$ that a connected bridgeless plane graph $G$ has a facial parity edge colouring with at most $\chi_{f p}^{\prime}(G)$ colours?

The number $\chi_{f p}^{\prime}(G)$ is called the facial parity chromatic index of $G$.

\section{Results}

Theorem 3.1. Let $G$ be a connected bridgeless plane graph. Then

$$
\chi_{f p}^{\prime}(G) \leq 92 .
$$

The proof uses the method of discharging. Let $G$ be a counterexample with minimal number of edges, then minimal number of 1 -faces, and then minimal number of 2 -faces. If $G$ is a single cycle of length $d, d \leq 5$, we use exactly $d$ colours. We consider this to be the first step of induction and call this case trivial.

First, we prove several structural properties of $G$.

We say that a face $\alpha$ is small if $1 \leq \operatorname{deg}(\alpha) \leq 44$ and a face $\beta$ is big if $\operatorname{deg}(\beta) \geq 45$.

\subsection{Reducible configurations}

We find such (forbidden) subgraphs $H$ of $G$ that the facial parity edge colouring of $G / H$ using at most 92 colours can be extended to a required colouring of $G$ using at most 92 
colours, which is a contradiction to $G$ being a counterexample. In the sequel, whenever we speak about an FPE colouring, we always mean an FPE colouring using at most 92 colours.

\subsubsection{1-faces}

Claim 3.2. Each vertex of $G$ is incident with at most one 1-face.

Proof. Let $v$ be a vertex incident with at least two 1-faces $\alpha_{1}$ and $\alpha_{2}$. If we split $v$ into two vertices $v_{1}$ and $v_{2}$ in such a way that $\alpha_{1}$ and $\alpha_{2}$ become a 2-face $\alpha$ and face-adjacency of all edges incident with $v$ is preserved, we obtain a graph $G^{\prime}$, see Figure 1 . It has the same number of edges as $G$, but fewer 1-faces. Thus, it is not a counterexample and we can find an FPE colouring $\varphi^{\prime}$ of $G^{\prime}$. It induces an edge colouring $\varphi$ of $G$ in a natural way. It is easy to see that $\varphi$ is an FPE colouring.
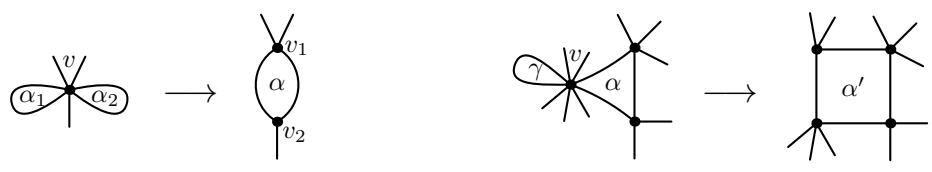

Figure 1: A vertex incident with at least two 1-faces can be split into two vertices, reducing the number of 1 -faces. Similarly, one can reduce a 1 -face and a $d$-face $(2 \leq d \leq 4)$ incident with the same vertex.

Claim 3.3. Each vertex of $G$ incident with a 1 -face is not incident with any other d-face for $2 \leq d \leq 4$.

Proof. We use the same reduction as in the proof of Claim 3.2. We split the vertex $v$ incident with a 1 -face $\gamma$ and a $d$-face $\alpha(2 \leq d \leq 4)$ in such a way that the faces $\alpha$ and $\gamma$ become a $(d+1)$-face $\alpha^{\prime}$ and face-adjacency of all edges incident with $v$ is preserved, see Figure 1 for illustration. Let the reduced graph be $G^{\prime}$. It has fewer 1 -faces than $G$, therefore, it has an FPE colouring $\varphi^{\prime}$. The face $\alpha^{\prime}$ has size at most five, therefore, its edges are coloured using $d+1$ different colours. Thus, the colouring $\varphi$ of $G$ induced by the colouring $\varphi^{\prime}$ of $G^{\prime}$ is an FPE colouring, too.

Claim 3.4. Each vertex of $G$ incident with a 2 -face is not incident with any $d$-face for $2 \leq d \leq 3$.

Proof. We use the same reduction as in the proofs of Claims 3.2 and 3.3. We omit the details.

\subsubsection{Small faces}

Claim 3.5. There are no two adjacent small faces in $G$.

Proof. Let $\alpha_{1}$ and $\alpha_{2}$ be two adjacent small faces in $G$.

If both $\alpha_{1}$ and $\alpha_{2}$ are 1 -faces, the graph consists of a single vertex and a loop; it has an FPE colouring using 1 colour. 
Let $\alpha_{1}$ be a 1 -face and $\alpha_{2}$ be a $d$-face, $d \geq 2$; let $e$ be the loop they share, see Figure 2 . Then the graph $G^{\prime}=G / e$ has fewer edges than $G$, therefore, it has an FPE colouring $\varphi^{\prime}$. Let $\alpha^{\prime}$ be a face in $G^{\prime}$ corresponding to $\alpha_{1}$ and $\alpha_{2}$ in $G$. Since $\alpha_{2}$ is a small face, at most 43 colours occur on the edges incident with $\alpha^{\prime}$. To extend the colouring $\varphi^{\prime}$ of $G^{\prime}$ to an FPE colouring of $G$, it suffices to colour the edge $e$ with any colour that does not occur on $\alpha^{\prime}$.

Let $\alpha_{1}$ and $\alpha_{2}$ be two small faces of size at least 2 and let $e$ be the edge they share, see Figure 2. The graph $G^{\prime}=G / e$ has fewer edges than $G$, therefore, it has an FPE colouring $\varphi^{\prime}$. Let $\alpha_{1}^{\prime}$ and $\alpha_{2}^{\prime}$ be the faces of $G^{\prime}$ corresponding to the faces $\alpha_{1}$ and $\alpha_{2}$ in $G$. (Since $\alpha_{1}$ and $\alpha_{2}$ are faces of size at least 2, the size of $\alpha_{1}^{\prime}$ and $\alpha_{2}^{\prime}$ is at least 1.)

Consider the set of colours different from the colours occurring on the edges of $\alpha_{1}^{\prime}$ and $\alpha_{2}^{\prime}$ (the colours admissible for the edge $e$ ). Since $\alpha_{1}$ and $\alpha_{2}$ are small, at most $2 \cdot(44-1)=86$ colours occur on the edges incident with $\alpha_{1}^{\prime}$ and $\alpha_{2}^{\prime}$. Hence, there is an admissible colour $c$. We can extend $\varphi^{\prime}$ to an FPE colouring $\varphi$ of $G$ by setting $\varphi(e)=c$.
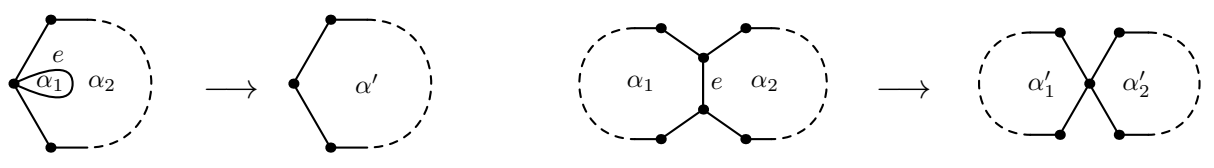

Figure 2: Two adjacent small faces form a reducible configuration: one can contract the edge they share.

Claim 3.6. Let $\beta$ be a big face adjacent to two small faces $\alpha_{1}$ and $\alpha_{2}$. Let $e_{i}$ be an edge incident with $\beta$ and $\alpha_{i}, i=1,2$. Then $e_{1}$ and $e_{2}$ are face-adjacent.

Proof. Let $e_{1}$ and $e_{2}$ not be face-adjacent. See Figure 3 for illustration. The graph $G^{\prime}=G /\left\{e_{1}, e_{2}\right\}$ has fewer edges than $G$, therefore, it has an FPE colouring $\varphi^{\prime}$. Let $\alpha_{1}^{\prime}$, $\alpha_{2}^{\prime}, \beta^{\prime}$ be the faces of $G^{\prime}$ corresponding to the faces $\alpha_{1}, \alpha_{2}, \beta$ in $G$, respectively.

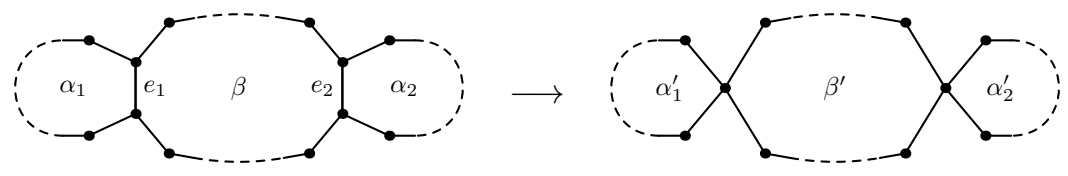

Figure 3: A big face $\beta$ adjacent to two different small faces $\alpha_{1}$ and $\alpha_{2}$ forms a reducible configuration unless the edges $e_{1}$ and $e_{2}$ are face-adjacent.

We extend the colouring $\varphi^{\prime}$ of $G^{\prime}$ to an FPE colouring of $G$ in the following way: Consider the set of colours different from the colours occurring on the edges of $\alpha_{1}^{\prime}$ and $\alpha_{2}^{\prime}$; also different from the colours occurring on the edges of $G^{\prime}$ corresponding to the edges of $G$ face-adjacent to $e_{1}$ and $e_{2}$. There are at least $92-2 \cdot(44-1)-4=2$ such colours, say $c_{1}$ and $c_{2}$. If at least one of them, say $c_{i}$, already occurs on $\beta^{\prime}$, we set $\varphi\left(e_{1}\right)=\varphi\left(e_{2}\right)=c_{i}$. If none of them occurs on $\beta^{\prime}$, we set $\varphi\left(e_{i}\right)=c_{i}, i=1,2$.

Claim 3.7. Each big face is adjacent to at most one 1-face.

Proof. It follows from Claims 3.2 and 3.6. 
Claim 3.8. Each big face is adjacent to at most two small faces.

Proof. Let a big face $\beta$ be adjacent to small faces $\alpha_{1}, \alpha_{2}$, and $\alpha_{3}$. Consider the edges that $\beta$ shares with $\alpha_{i}, i=1,2,3$. It is easy to see that there must be a pair of edges $e_{i}$ and $e_{j}$, incident to $\alpha_{i}$ and $\alpha_{j}$, respectively $(i \neq j)$, which are not face-adjacent. It is a contradiction with Claim 3.6.

\subsubsection{Chains of 2-vertices}

Claim 3.9. There is no chain consisting of at least 5 consecutive 2-vertices in G.

Proof. Let $v_{0} e_{0} v_{1} e_{1} \ldots v_{p} e_{p} v_{p+1}$ be a chain consisting of $p$ vertices $v_{1}, \ldots, v_{p}$ of degree 2 , where $p \geq 5$. The graph $G^{\prime}=G /\left\{e_{1}, e_{2}, e_{3}, e_{4}\right\}$ has an FPE colouring $\varphi^{\prime}$. Let $e_{0}^{\prime}$ and $e_{5}^{\prime}$ be the edges in $G^{\prime}$ corresponding to the edges $e_{0}$ and $e_{5}$ in $G$. Let $\varphi^{\prime}\left(e_{0}^{\prime}\right)=c_{1}$ and $\varphi^{\prime}\left(e_{5}^{\prime}\right)=c_{2}$. The FPE colouring $\varphi^{\prime}$ of $G^{\prime}$ can be extended to an FPE colouring $\varphi$ of $G$ by setting $\varphi\left(e_{2}\right)=\varphi\left(e_{4}\right)=c_{1}$ and $\varphi\left(e_{1}\right)=\varphi\left(e_{3}\right)=c_{2}$, see Figure 4 .

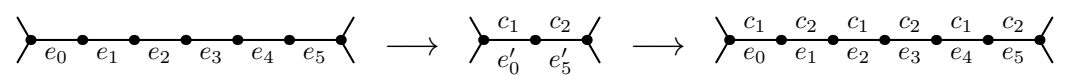

Figure 4: A chain of (at least) five 2-vertices is a reducible configuration.

A $d$-face $\alpha$ is hanging on a vertex $v$, if all vertices incident with $\alpha$ are 2-vertices except for the vertex $v$. By Claim 3.9 we have $d \leq 5$. (If $\operatorname{deg}(v)=2$, the graph $G$ consists of a single cycle of length at most 5 , which is the trivial case).

We colour the vertices of $G$ with black, blue and white colour in the following way:

Let all 2-vertices be black, all 3 -vertices be blue and all $k$-vertices for $k \geq 6$ be white.

A 4 -vertex $v$ is black if there is a face hanging on it, else it is white. See Figure 5 for illustration of all types of black vertices.

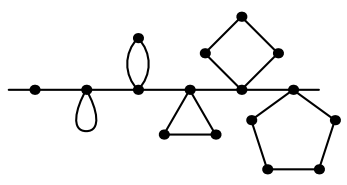

Figure 5: A vertex is black, if it is a 2-vertex, or a 4-vertex with a hanging face.

A 5-vertex $v$ is blue, if there is a face hanging on it, else it is white.

Observe that any 2-vertex $v$ is incident with two faces. We say $v$ is $b a d$ for both faces it is incident with. Any black 4 -vertex $v$ is incident with a small face $\alpha$ of size at most 5 and two other faces. The face $\beta$ adjacent to $\alpha$ must be big (see Claim 3.5); the vertex $v$ occurs twice on the facial walk of $\beta$. The other face $\gamma$ can be big or small. We say $v$ is bad for the face $\gamma$.

Claim 3.10. For any face $\alpha$, there is no chain of at least 5 consecutive vertices bad for $\alpha$. Each chain of bad vertices contains at most one bad 4-vertex. 
Proof. Let $v_{0} e_{0} v_{1} e_{1} v_{2} e_{2} v_{3} e_{3} v_{4} e_{4} v_{5} e_{5} v_{6}$ be a subpath of the facial walk of $\alpha$ containing 5 vertices $v_{1}, \ldots, v_{5}$ bad for $\alpha$. It is easy to see that all the edges $e_{0}, \ldots, e_{5}$ are incident with the same face $\beta(\beta \neq \alpha)$. If at least two of $v_{1}, \ldots, v_{5}$ are bad 4 -vertices, we come to a contradiction with Claim 3.5 or with Claim 3.6. If none of them is a 4 -vertex, we are in the case of Claim 3.9. If precisely one of them is a 4 -vertex, say $v_{i}$, it is incident with a small face $\gamma$ of size $d \leq 5$.

The graph $G^{\prime}=G /\left\{e_{1}, e_{2}, e_{3}, e_{4}\right\}$ has an FPE colouring $\varphi^{\prime}$. Let $e_{0}^{\prime}$ and $e_{5}^{\prime}$ be the edges in $G^{\prime}$ corresponding to the edges $e_{0}$ and $e_{5}$ in $G$; let $\alpha^{\prime}, \beta^{\prime}, \gamma^{\prime}$ be the faces in $G^{\prime}$ corresponding to the faces $\alpha, \beta, \gamma$ in $G$; let $v^{\prime}$ be the vertex in $G^{\prime}$ corresponding to $v_{1}, \ldots, v_{5}$ in $G$. Let $\varphi^{\prime}\left(e_{0}^{\prime}\right)=c_{1}$ and $\varphi^{\prime}\left(e_{5}^{\prime}\right)=c_{2}$. Since $e_{0}^{\prime}$ and $e_{5}^{\prime}$ are face-adjacent in $G^{\prime}, c_{1} \neq c_{2}$. To extend $\varphi^{\prime}$ to an FPE colouring of $G$, we proceed in the following way:

If $i=1,3$, or 5 , we simply set $\varphi\left(e_{2}\right)=\varphi\left(e_{4}\right)=c_{1}$ and $\varphi\left(e_{1}\right)=\varphi\left(e_{3}\right)=c_{2}$.

If $i=2$ or $i=4$, we set $\varphi\left(e_{2}\right)=\varphi\left(e_{4}\right)=c_{1}$ and $\varphi\left(e_{1}\right)=\varphi\left(e_{3}\right)=c_{2}$ and switch the order of colours of edges incident with $\gamma^{\prime}$, see Figure 6 for illustration (here the small face $\gamma$ is a bigon).
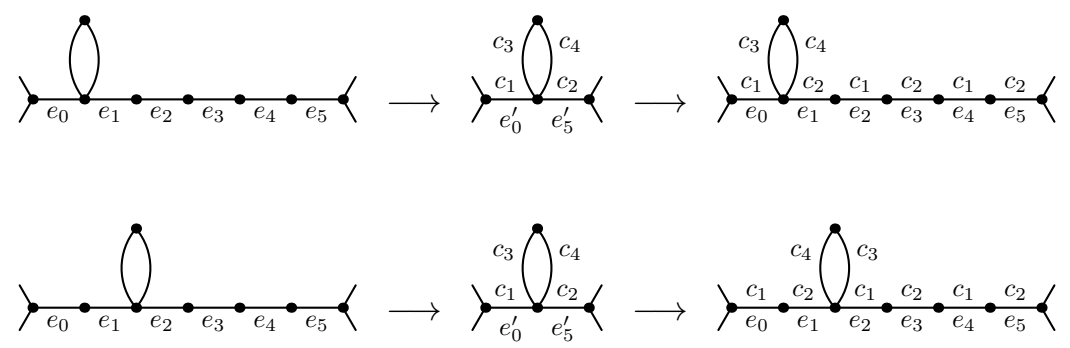

Figure 6: A chain of (at least) five bad black vertices is a reducible configuration as well.

Claim 3.11. Let $v$ be a black vertex bad for a small face $\alpha$. If $v$ is a 4-vertex, then the face hanging on it is a 1-face.

Proof. Let $v$ be a black 4-vertex, let $\gamma$ be the face hanging on $v$ of size at least 2 and let $e_{1}$ and $e_{2}$ be the edges of $\alpha$ incident with $v$. It is easy to see that the edges $e_{1}$ and $e_{2}$ are incident with the same big face, say $\beta$. See Figure 7 for illustration.

There is an edge $e_{\gamma}$ incident with $\gamma$ and $\beta$, which is not face-adjacent to $e_{\alpha} \in\left\{e_{1}, e_{2}\right\}$, which is a contradiction with Claim 3.6.
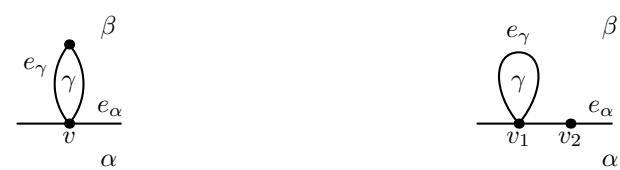

Figure 7: Reducible pairs of edges $\left(e_{\gamma}\right.$ and $\left.e_{\alpha}\right)$. For details see Claims 3.11 and 3.12. 
Claim 3.12. Let $\alpha$ be a small face sharing at least two bad black vertices with a big face $\beta$. Then all the bad black vertices incident with $\alpha$ and $\beta$ are 2 -vertices.

Proof. Let $v_{1}$ and $v_{2}$ be black vertices incident with $\alpha$ and $\beta$. If $v_{1}$ is a 4 -vertex, it is bad for $\alpha$, therefore, there is a small face $\gamma$ hanging on $v_{1}$, adjacent to $\beta$. By Claim 3.11, the face $\gamma$ is a 1-face. Thus, we can find an edge (a loop) $e_{\gamma}$ incident with $\gamma$ and $\beta$ and an edge $e_{\alpha}$ incident with $\alpha$ and $\beta$, which are not face-adjacent. This is a contradiction with Claim 3.6. See Figure 7 for illustration.

Claim 3.13. Let $v$ be a black vertex bad for a small $d$-face $\alpha, d \in\{2,3,4\}$. Then $v$ is a 2-vertex.

Proof. Let $v$ be a black 4-vertex, let $\gamma$ be the face hanging on $v$. By Claim 3.11, the face $\gamma$ is a 1 -face. On the other hand, by Claim 3.3, the face $\gamma$ cannot be a 1 -face, which is a contradiction.

Claim 3.14. If a big face $\beta$ shares a 2-vertex $v$ with a small face $\alpha$, then $\beta$ is not adjacent to any other small face.

Proof. It follows immediately from Claim 3.6.

Claim 3.15. Let $\gamma$ be a $d$-face, $d \in\{2,3,4,5\}$, hanging on a vertex $v$, adjacent to a big face $\beta$. Then $\beta$ is not adjacent to any other small face.

Proof. It follows immediately from Claim 3.14.

\subsection{Discharging rules}

If $G$ is a minimal counterexample then it contains no reducible configuration. Let the initial charge of each vertex be $\psi(v)=\operatorname{deg}(v)-6$ and the initial charge of each face be $\psi(\alpha)=2 \operatorname{deg}(\alpha)-6$. From Euler's formula we can easily derive that

$$
\sum_{\alpha \in F}(2 \operatorname{deg}(\alpha)-6)+\sum_{v \in V}(\operatorname{deg}(v)-6)=-12 .
$$

It is obvious that all the negative charge is in the vertices of degree $2,3,4$, and 5 and in the faces of size 1 and 2 .

Rule 1: Let $\beta$ be a big face.

- If $\beta$ is adjacent to a single small face $\alpha$, it sends 3 units of charge to $\alpha$.

- If $\beta$ is adjacent to two small faces $\alpha_{1}$ and $\alpha_{2}$, such that $\operatorname{deg}\left(\alpha_{1}\right) \leq \operatorname{deg}\left(\alpha_{2}\right)$, it sends 2 units of charge to $\alpha_{1}$ and 1 unit of charge to $\alpha_{2}$. (If $\operatorname{deg}\left(\alpha_{1}\right)=\operatorname{deg}\left(\alpha_{2}\right)$, it is decided arbitrarily.)

Rule 2: Let $\beta$ be a big face.

- It sends 2 units of charge to any black vertex bad for $\beta$.

- It sends 1 unit of charge to any other black, blue, or white vertex incident with $\beta$. (Multiply incident vertices are considered as different.)

Rule 3: Let $\alpha$ be a small face. 
- It sends 2 units of charge to any black vertex bad for $\alpha$.

- It sends 1 unit of charge to any other black or blue vertex incident with $\alpha$.

Rule 4: Let $v$ be a black 4-vertex.

- It sends 2 units of charge to the incident small hanging face $\gamma$.

Rule 5: Let $v$ be a blue 5-vertex.

- It sends 2 units of charge to the incident small hanging face $\gamma$.

Rule 6: Let $v$ be a $k$-vertex, $k \geq 6$.

- It sends 2 units of charge to any incident small hanging face $\gamma$.

\subsection{Analysis of the discharging process}

\subsubsection{Vertices}

Every 2-vertex is black and bad for both faces incident with it, hence it receives 2 units of charge from both incident faces (Rules 2 and 3). Its new charge is $-4+2+2=0$.

Every 3 -vertex is blue, hence it receives 1 unit of charge from all the three incident faces (Rules 2 and 3). Its new charge is $-3+3 \cdot 1=0$.

Every black 4-vertex $v$ receives 2 units of charge from the face it is bad for (Rules 2 and 3 ) and $2 \cdot 1$ units of charge from the doubly-incident big face $\beta$ (Rule 2). It sends 2 units of charge to the hanging face $\gamma$ (Rule 4). The new charge of $v$ is $-2+2+2 \cdot 1-2=0$.

Every white 4 -vertex is incident with at least 2 big faces (see Claim 3.5), therefore, its new charge is at least $-2+2 \cdot 1=0$.

Every blue 5 -vertex $v$ is incident with a hanging face $\gamma$, doubly-incident with a big face $\beta$ and incident with two more faces $\alpha_{1}$ and $\alpha_{2}$. Therefore, $v$ receives $4 \cdot 1$ units of charge from the incident faces (Rules 2 and 3). It sends 2 units of charge to $\gamma$ (Rule 5). Therefore, the new charge of $v$ is $-1+4 \cdot 1-2=1$.

Every white 5 -vertex is incident with at least 3 big faces (see Claim 3.5), therefore, its new charge is at least $-1+3 \cdot 1=2$.

Every (white) $k$-vertex $v, k \geq 6$, has non-negative initial charge. It receives charge from big faces (Rule 2) and sends charge to the hanging faces $\gamma_{1}, \ldots, \gamma_{r}$ (Rule 6). For each hanging face $\gamma_{i}$, the adjacent big face $\beta_{i}$ is doubly-incident to $v$. The faces $\beta_{i}$ and $\beta_{j}$ are different for different $\gamma_{i}$ and $\gamma_{j}$ (see Claims 3.6 and 3.7). Therefore, the new charge of $v$ is at least $r \cdot(2 \cdot 1-2)=0$.

\subsubsection{1-faces}

Let $\gamma$ be a 1-face. It is adjacent to a big face $\beta$ and it is hanging on a vertex $v$. The face $\beta$ is adjacent to at most one 1-face (Claim 3.7). Therefore, it sends at least 2 units of charge to the face $\gamma$ (Rule 1).

If the vertex $v$ is a 2-vertex, we get the trivial graph, which is not a counterexample. If the vertex $v$ is a 3 -vertex, the third edge incident with $v$ is a bridge in $G$, which is not allowed. Therefore, $\operatorname{deg}(v) \geq 4$ and the vertex $v$ sends 2 units of charge to the face $\gamma$ (Rules $4-6$ ). The new charge of $\gamma$ is at least $-4+2+2=0$. 


\subsubsection{2-faces}

Let $\alpha$ be a 2-face. Its initial charge is -2 . Let $v_{1}$ and $v_{2}$ be the vertices incident with $\alpha$. The face $\alpha$ is adjacent to at most two faces, which must be big (see Claim 3.5). Consider the number of black vertices bad for $\alpha$. Note that by Claim 3.13 each such vertex is a 2 -vertex. See Figure 8 for illustration.

1. Let both $v_{1}$ and $v_{2}$ be black and bad. Then the graph $G$ consists of a single cycle on two vertices, which is not a counterexample.

2. Let $v_{1}$ be a black 2 -vertex. Then $\alpha$ is adjacent to a single big face $\beta$. The big face $\beta$ is not adjacent to any other small face (see Claim 3.14). Therefore, $\beta$ sends 3 units of charge to $\alpha$ (Rule 1 ). The face $\alpha$ sends 2 units of charge to $v_{1}$ and at most 1 unit of charge to $v_{2}$ (Rule 3). On the other hand, $\alpha$ is hanging on $v_{2}$, therefore, it receives 2 units of charge from $v_{2}$ (Rules $4-6$ ). Note that $v_{2}$ cannot be a 3 -vertex, otherwise there would be a bridge in $G$. The new charge of $\alpha$ is at least $-2-2-1+2+3=0$.

3. Let none of $v_{1}$ and $v_{2}$ be black and bad. Consider the number of faces adjacent to $\alpha$. If $\alpha$ is adjacent to a single big face $\beta$, then $\beta$ is not adjacent to any other small face. Therefore, $\beta$ sends 3 units of charge to $\alpha$. Moreover, in this case none of $v_{1}$ and $v_{2}$ can be neither black nor blue. The new charge of $\alpha$ is $-2+3=1$.

If $\alpha$ is adjacent to two big faces $\beta_{1}$ and $\beta_{2}$, the face $\alpha$ sends at most $2 \cdot 1$ units of charge to $v_{1}$ and $v_{2}$. The big face $\beta_{i}, i=1,2$, is not adjacent to any other small face of size at most 2 (see Claims 3.3, 3.4, and 3.6). Therefore, by Rule 1, the face $\beta_{i}, i=1,2$, sends at least 2 units of charge to $\alpha$. The new charge of $\alpha$ is at least $-2-2 \cdot 1+2 \cdot 2=0$.
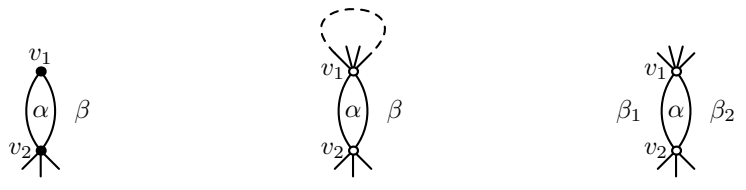

Figure 8: Different possible neighbourhoods of a 2-face $\alpha$.

\subsubsection{3-faces}

Let $\alpha$ be a 3 -face. Its initial charge is 0 . Let $v_{1}, v_{2}, v_{3}$ be the vertices incident with $\alpha$. (They do not have to be pairwise different.) Consider the number of black vertices bad for $\alpha$. Note that by Claim 3.13 each such vertex is a 2-vertex. See Figure 9 for illustration.

1. Let all the three vertices $v_{1}, v_{2}, v_{3}$ be black and bad. Then the graph $G$ consists of a single cycle on three vertices, which is not a counterexample.

2. Let $v_{2}$ and $v_{3}$ be black and bad. Then all the three edges of $\alpha$ are incident with the same big face $\beta$ and $\alpha$ is hanging on $v_{1}$. Then by Claim 3.15 the face $\beta$ sends 3 units of charge to $\alpha$ (Rule 1). The face $\alpha$ then sends $2 \cdot 2$ units of charge to the 2 -vertices $v_{2}, v_{3}$ and at most 1 unit of charge to the vertex $v_{1}$ (Rule 3 ). If the vertex $v_{1}$ is a 3 -vertex, the third edge incident with it is a bridge in $G$. Therefore 
$\operatorname{deg}\left(v_{1}\right) \geq 4$ and $v_{1}$ sends 2 units of charge to $\alpha$ (Rules $\left.4-6\right)$. The new charge of $\alpha$ is $3-2 \cdot 2-1+2=0$.

3. Let $v_{3}$ be black and bad. Let $\beta_{1}$ be the big face incident with the edge $v_{1} v_{2}$ and $\beta_{2}$ be the big face incident with the edges $v_{2} v_{3}$ and $v_{3} v_{1}$.

The face $\beta_{2}$ sends 3 units of charge to $\alpha, \beta_{1}$ sends at least 1 unit of charge to $\alpha$. The face $\alpha$ then sends 2 units of charge to $v_{3}$ and at most 1 unit of charge to $v_{1}$ and $v_{2}$. The new charge of $\alpha$ is at least $3+1-2-2 \cdot 1=0$.

4. Let none of the vertices $v_{1}, v_{2}, v_{3}$ be black and bad. Consider the number of faces adjacent to $\alpha$.

If there are three different faces adjacent to $\alpha$ (they must be big, see Claim 3.5) then the face $\alpha$ receives at least 1 unit of charge from each of them and sends at most 1 unit of charge to each incident vertex. Hence, the new charge of $\alpha$ is at least 0 .

If there is a big face $\beta$ sharing at least two edges with $\alpha$, these edges are not faceadjacent in $\beta$. Therefore, $\beta$ is not adjacent to any other small face, thus, it sends 3 units of charge to $\alpha$. The new charge of $\alpha$ is non-negative again.
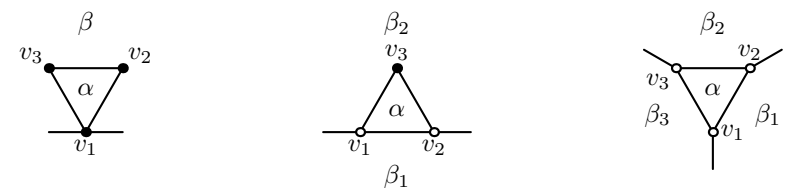

Figure 9: Different possible neighbourhoods of a 3-face $\alpha$.

\subsubsection{4-faces}

Let $\alpha$ be a 4 -face. Its initial charge is 2 . Let $v_{1}, v_{2}, v_{3}, v_{4}$ be the vertices incident with $\alpha$. (They do not have to be pairwise different.) Consider the number of black vertices bad for $\alpha$. Note that by Claim 3.13 each such vertex is a 2-vertex. See Figure 10 for illustration.

1. Let all the four vertices $v_{1}, v_{2}, v_{3}, v_{4}$ be black and bad. Then the graph $G$ consists of a single cycle on four vertices, which is not a counterexample.

2. Let $v_{1}, v_{2}$, and $v_{3}$ be black and bad. Then all the four edges of $\alpha$ are incident with the same big face $\beta$ and $\alpha$ is hanging on $v_{4}$. Hence, $\beta$ sends 3 units of charge to $\alpha$ (Rule 1 ). The face $\alpha$ then sends $3 \cdot 2$ units of charge to the 2 -vertices $v_{1}, v_{2}, v_{3}$ and at most 1 unit of charge to $v_{4}$ (Rule 3 ). If the vertex $v_{4}$ is a 3 -vertex, the third edge incident with it is a bridge in $G$. Therefore, $\operatorname{deg}\left(v_{4}\right) \geq 4$ and $v_{4}$ sends 2 units of charge to $\alpha$ (Rules $4-6$ ). The new charge of $\alpha$ is $2+3-3 \cdot 2-1+2=0$.

3. Let $v_{1}$ and $v_{3}$ be black and bad. Let $\beta_{1}$ be the big face incident with $v_{1}$, let $\beta_{2}$ be the big face incident with $v_{3}$.

If $\beta_{1} \neq \beta_{2}$, both $\beta_{1}$ and $\beta_{2}$ send 3 units of charge to $\alpha$. The face $\alpha$ then sends 2 units of charge to the vertices $v_{1}$ and $v_{3}$ and at most 1 unit of charge to the vertices $v_{2}$ and $v_{4}$. The new charge of $\alpha$ is at least $2+2 \cdot 3-2 \cdot 2-2 \cdot 1=2$. If $\beta_{1}=\beta_{2}$, then $v_{2}$ and $v_{4}$ are not blue, and the new charge of $\alpha$ is at least $2+3-2 \cdot 2=1$. 
4. Let $v_{1}$ and $v_{2}$ be black and bad. Let $\beta_{1}$ be the big face incident with the edge $v_{3} v_{4}$ and $\beta_{2}$ be the big face incident with the vertices $v_{1}$ and $v_{2}$. If $\beta_{1} \neq \beta_{2}, \beta_{2}$ sends 3 units of charge to $\alpha$ and $\beta_{1}$ sends at least 1 unit of charge to $\alpha$. The face $\alpha$ then sends 2 units of charge both to $v_{1}$ and $v_{2}$ and at most 1 unit of charge to $v_{3}$ and $v_{4}$. The new charge of $\alpha$ is at least $2+3+1-2 \cdot 2-2 \cdot 1=0$. If $\beta_{1}=\beta_{2}$, then $v_{3}$ and $v_{4}$ are not blue, and the new charge of $\alpha$ is at least $2+3-2 \cdot 2=1$.

5. Let $v_{1}$ be black and bad. The big face $\beta_{1}$ incident with $v_{1}$ sends 3 units of charge to $\alpha$. The face $\alpha$ then sends 2 units of charge to $v_{1}$ and at most 1 unit of charge to $v_{2}, v_{3}$, and $v_{4}$. The new charge of $\alpha$ is at least $2+3-2-3 \cdot 1=0$.

6. Let no black and bad vertex be incident with $\alpha$. Then the big faces adjacent to $\alpha$ send together at least 2 units of charge (if there was only one big face, it would send 3 units of charge), and $\alpha$ sends at most 1 unit of charge to each incident vertex. The new charge of $\alpha$ is at least $2+2-4=0$.
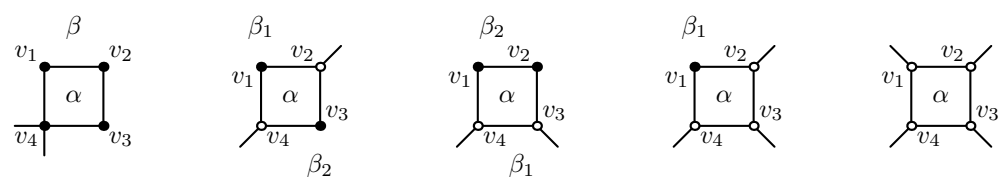

Figure 10: Different possible neighbourhoods of a 4-face $\alpha$.

\subsubsection{5-faces}

Let $\alpha$ be a 5 -face. Its initial charge is 4 . Let $v_{1}, v_{2}, v_{3}, v_{4}, v_{5}$ be the vertices incident with $\alpha$. (They do not have to be pairwise different.) Consider the number of black vertices bad for $\alpha$. Note that by Claim 3.11 each such vertex is either a 2 -vertex or a 4 -vertex with a hanging 1-face. See Figure 11 for illustration.

1. Let all the five vertices $v_{1}, v_{2}, v_{3}, v_{4}, v_{5}$ be black and bad. Then the graph $G$ contains only 5 vertices, hence, it is not a counterexample.

2. Let $v_{1}, v_{2}, v_{3}$, and $v_{4}$ be black and bad. From Claim 3.6 it follows that none of them is incident with a 1 -face. Then all the five edges of $\alpha$ are incident with the same big face $\beta$ and $\alpha$ is hanging on $v_{5}$. Hence, $\beta$ sends 3 units of charge to $\alpha$ (Rule 1 ). The face $\alpha$ then sends $4 \cdot 2$ units of charge to the 2 -vertices $v_{1}, v_{2}, v_{3}$, and $v_{4}$ (Rule 3 ) and at most 1 unit of charge to the vertex $v_{5}$ (Rule 3 ). If the vertex $v_{5}$ is a 3 -vertex, the third edge incident with it is a bridge in $G$. Therefore $\operatorname{deg}\left(v_{5}\right) \geq 4$ and $v_{5}$ sends 2 units of charge to $\alpha$ (Rules $4-6$ ). The new charge of $\alpha$ is $4+3-4 \cdot 2-1+2=0$.

3 . Let $v_{1}, v_{2}$, and $v_{3}$ be black and bad. Let $\beta_{1}$ be the big face incident with the vertices $v_{1}, v_{2}$, and $v_{3}$ and $\beta_{2}$ be the big face incident with the edge $v_{4} v_{5}$. By Claim 3.12 $v_{1}, v_{2}$, and $v_{3}$ are 2-vertices. If $\beta_{1} \neq \beta_{2}, \beta_{1}$ sends 3 units of charge to $\alpha$ and $\beta_{2}$ sends at least 1 unit of charge to $\alpha$. The face $\alpha$ then sends 2 units of charge to $v_{1}, v_{2}$, and $v_{3}$ and at most 1 unit of charge to $v_{4}$ and $v_{5}$. The new charge of $\alpha$ is at least $4+3+1-3 \cdot 2-2 \cdot 1=0$. If $\beta_{1}=\beta_{2}$, then $v_{4}$ and $v_{5}$ are not blue, and the new charge of $\alpha$ is at least $4+3-3 \cdot 2=1$. 
4. Let $v_{1}, v_{2}$, and $v_{4}$ be black and bad. Let $\beta_{1}$ be the big face incident with $v_{1}$ and $v_{2}$, let $\beta_{2}$ be the big face incident with $v_{4}$. By Claim $3.12 v_{1}$ and $v_{2}$ are 2-vertices, thus $\beta_{1}$ sends 3 units of charge to $\alpha$.

If $\beta_{1} \neq \beta_{2}$, then $\beta_{2}$ sends at least 1 unit of charge to $\alpha$. The face $\alpha$ then sends 2 units of charge to the vertices $v_{1}, v_{2}$, and $v_{4}$ and at most 1 unit of charge to the vertices $v_{3}$ and $v_{5}$. The new charge of $\alpha$ is at least $4+3+1-3 \cdot 2-2 \cdot 1=0$. If $\beta_{1}=\beta_{2}$, then $v_{3}$ and $v_{5}$ are not blue, and the new charge of $\alpha$ is $4+3-3 \cdot 2=1$.

5. Let $v_{1}$ and $v_{2}$ be black and bad. Let $\beta_{1}$ be the big face incident with the vertices $v_{1}$ and $v_{2}$. The face $\beta_{1}$ sends 3 units of charge to $\alpha$. The face $\alpha$ then sends 2 units of charge both to $v_{1}$ and $v_{2}$ and at most 1 unit of charge to $v_{3}, v_{4}$, and $v_{5}$. The new charge of $\alpha$ is at least $4+3-2 \cdot 2-3 \cdot 1=0$.

6. Let $v_{1}$ and $v_{3}$ be black and bad. Let $\beta_{1}$ be the big face incident with $v_{1}, \beta_{2}$ be the big face incident with $v_{3}$, and $\beta_{3}$ be the big face incident with the edge $v_{4} v_{5}$. If $\beta_{1}, \beta_{2}$, and $\beta_{3}$ are three different faces, the new charge of $\alpha$ is at least $4+3-2 \cdot 2-3 \cdot 1=0$. If two of them coincide, then at least one of the vertices $v_{2}, v_{4}$, and $v_{5}$ is not blue and the new charge of $\alpha$ is at least $4+2-2 \cdot 2-2 \cdot 1=0$. If $\beta_{1}=\beta_{2}=\beta_{3}$, then $v_{2}, v_{4}$, and $v_{5}$ are not blue and the new charge of $\alpha$ is at least $4+1-2 \cdot 2=1$.

7. Let $v_{1}$ be black and bad. Then the big faces adjacent to $\alpha$ send together at least 2 units of charge (if there was only one big face, it would send 3 units of charge). The new charge of $\alpha$ is at least $4+2-2-4 \cdot 1=0$.

8. Let no black and bad vertex be incident with $\alpha$. Then the big faces adjacent to $\alpha$ send together at least 1 unit of charge. The new charge of $\alpha$ is at least $4+1-5=0$.

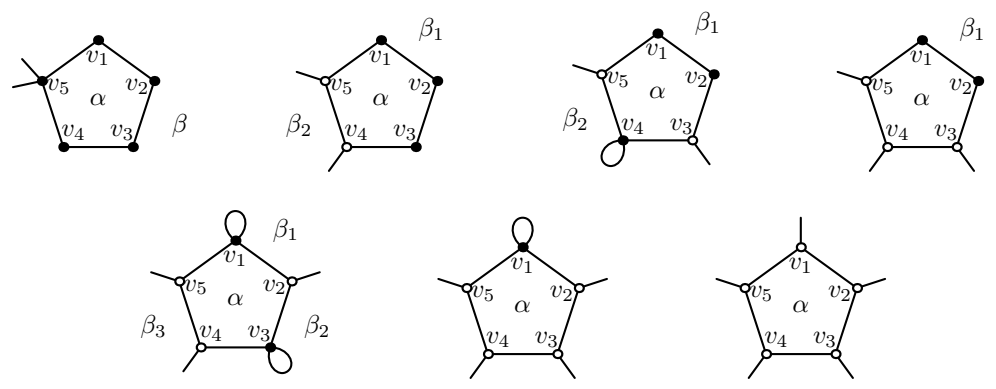

Figure 11: Different possible neighbourhoods of a 5 -face $\alpha$.

\subsubsection{Small faces of size at least 6}

Let $\alpha$ be a $d$-face, $6 \leq d \leq 44$. Its initial charge is $2 d-6$. Let $v_{1}, \ldots, v_{d}$ be the vertices incident with $\alpha$. (They do not have to be pairwise different.) Consider the black vertices incident with $\alpha$. Let $v_{i}$ be a black 4 -vertex. It cannot be good for $\alpha$, since no two small faces are adjacent (see Claim 3.5). Therefore, each black 4-vertex is bad for $\alpha$. By Claim 3.10 at most $d-2$ vertices incident with $\alpha$ are bad. Let $k \leq d-2$ be the number of black vertices incident with $\alpha$. We can divide the facial walk of $\alpha$ into $d-k \geq 2$ parts, each beginning and ending in a blue or white vertex, each incident with $\alpha$ and a big face $\beta_{i}$, 
$i \in\{1, \ldots, d-k\}$. Each of these big faces sends at least 1 unit of charge to $\alpha$. (If $\beta_{i}=\beta_{j}$ for some $1 \leq i<j \leq d-k$, the face $\beta_{i}$ cannot be adjacent to another small face but $\alpha$, therefore, it sends 3 units to $\alpha$, which is even more than what two different big faces would send.)

The face $\alpha$ then sends 2 units of charge to each of the $k$ incident black vertices, and at most 1 unit of charge to each of the other incident vertices. Together, the new charge of $\alpha$ is at least

$$
2 d-6+(d-k) \cdot 1-k \cdot 2-(d-k) \cdot 1=2(d-k)-6 .
$$

If $d-k \geq 3$, the new charge of $\alpha$ is non-negative.

Let $d-k=2$. It means there are only two vertices which are not black. Since $d \geq 6$, at least one big face $\beta$ shares at least 2 black vertices with $\alpha$, say $v_{1}$ and $v_{2}$. By Claim 3.10 at least one from $v_{1}$ and $v_{2}$ is a 2 -vertex, hence, by Claim 3.14 the face $\beta$ sends 3 units of charge to $\alpha$. The new charge of $\alpha$ is therefore at least

$$
2 d-6+3+1-(d-2) \cdot 2-2 \cdot 1=0 .
$$

\subsubsection{Big faces}

Let $\beta$ be a $d$-face, $d \geq 45$. Its initial charge is $2 d-6$. It sends 3 units of charge to the small faces it is adjacent to (Rule 1). It sends 2 units of charge to all bad black vertices; 1 unit of charge to all other vertices. Let $k$ be the number of black vertices bad for $\beta$. By Claim $3.10, k \leq \frac{4}{5} \cdot d$. The new charge of $\beta$ is therefore at least

$$
2 d-6-3-k \cdot 2-(d-k) \cdot 1=d-k-9 \geq d-\frac{4 d}{5}-9=\frac{d}{5}-9=\frac{d-45}{5} \geq 0 .
$$

The new charge of all elements of the graph is non-negative, but the sum of all the charges is -12 , which is a contradiction. This contradiction implies that the minimum counterexample does not exist.

\section{Acknowledgments}

This work was supported by Slovak Research and Development Agency under contract No. APVV-0007-07. Support of Slovak VEGA Grant 1/0428/10 is acknowledged as well. Institute for Theoretical Computer Science (ITI) is supported by Ministry of Education of the Czech Republic as project 1M0545.

The authors thank the referees for many helpful comments and suggestions.

\section{References}

[1] D. P. Bunde, K. Milans, D. B. West and H. Wu, Optimal strong parity edge-coloring of complete graphs, Combinatorica 28 (2008), 625-632.

[2] D. P. Bunde, K. Milans, D. B. West and H. Wu, Parity and strong parity edge-coloring of graphs, Congressus Numerantium 187 (2007), 193-213.

[3] J. Czap and S. Jendrol, Colouring vertices of plane graphs under restrictions given by faces, Discussiones Math. Graph Theory 29 (2009), 521-543.

[4] T. Mátrai, Covering the edges of a graph by three odd subgraphs, J. Graph Theory 53 (2006), 75-82. 
[5] L. Pyber, Covering the edges of a graph by ..., Sets, Graphs and Numbers, Colloquia Mathematica Societatis János Bolyai 60 (1991), 583-610.

[6] V. G. Vizing, On an estimate of the chromatic class of a p-graph, Diskret. Analiz 3 (1964), 25-30. 